\title{
Advection-dominated accretion: Self-similarity and bipolar outflows
}

\section{Citation}

Narayan, Ramesh, and Insu Yi. 1995. "Advection-Dominated Accretion: Self-Similarity and Bipolar Outflows." The Astrophysical Journal 444 (May): 231. https://doi.org/10.1086/175599.

\section{Permanent link}

http://nrs.harvard.edu/urn-3:HUL.InstRepos:41384895

\section{Terms of Use}

This article was downloaded from Harvard University's DASH repository, and is made available under the terms and conditions applicable to Other Posted Material, as set forth at http:// nrs.harvard.edu/urn-3:HUL.InstRepos:dash.current.terms-of-use\#LAA

\section{Share Your Story}

The Harvard community has made this article openly available.

Please share how this access benefits you. Submit a story.

\section{Accessibility}




\title{
Advection-Dominated Accretion: Self-Similarity and Bipolar Outflows
}

\author{
Ramesh Narayan and Insu Yi \\ Harvard-Smithsonian Center for Astrophysics \\ 60 Garden Street, Cambridge, MA 02138
}

\begin{abstract}
We consider axisymmetric viscous accretion flows where a fraction $f$ of the viscously dissipated energy is stored in the accreting gas as entropy and a fraction $1-f$ is radiated. Assuming $\alpha$-viscosity we obtain a two-parameter family of self-similar solutions. Very few such exact self-consistent solutions are known for viscous differentially rotating flows. When the parameter $f$ is small, that is when there is very little advection, our solutions resemble standard thin accretion disks in many respects except that they have a hot tenuous corona above the disk. In the opposite advection-dominated limit, when $f \rightarrow 1$, the solutions approach nearly spherical accretion. The gas is almost at virial temperature, rotates at much below the Keplerian rate, and the flow is much more akin to Bondi accretion than to disk accretion. None of the solutions have funnels.

We compare our exact self-similar solutions with approximate solutions which had been previously obtained using a height-integrated system of equations. We find that various dynamical variables such as the radial velocity, angular velocity and sound speed estimated from the approximate solutions agree very well with the corresponding spherically averaged quantities in the exact solutions. We conclude that the height-integration approximation is an excellent one for a wide range of accretion conditions, including nearly spherical flows, provided the equations are interpreted as spherical averages.

We find that the Bernoulli parameter is positive in all our solutions, especially close to the rotation axis. This effect is produced by viscous transport of energy from small to large radii and from the equator to the poles. In addition, all the solutions are convectively unstable and the convection is especially important near the rotation axis. For both reasons, we suggest that a bipolar outflow will develop along the axis of these flows, fed by material from the surface layers of the equatorial inflow.
\end{abstract}

Subject headings: accretion, accretion disks: black hole physics: hydrodynamics 


\section{Introduction}

In a previous paper (Narayan \& Yi 1994, hereafter NY) we discussed the potential importance of advection effects in accretion flows. We showed that, both at very low and very high optical depths, the energy released through viscous stresses in an accretion disk may be trapped within the accreting gas. Most of the energy then is advected with the flow as stored entropy. Such advectiondominated flows have been found in models of boundary layers in cataclysmic variables at low accretion rates (Narayan \& Popham 1993) and in models of pre-main sequence stars such as the FU Orionis systems at very high accretion rates (Popham et al 1993). Advection-dominated conditions may also occur in the inner parts of disks around neutron stars and black holes.

In the analysis presented in NY, we integrated the flow equations in the "vertical" direction, i.e. parallel to the rotation axis. Making the usual assumptions of steady state, axisymmetry, and $\alpha$-viscosity, we obtained a set of ordinary differential equations for the gas variables as a function of the cylindrical radius $R$. We showed that these equations have an exact self-similar solution where all variables have power-law dependences on $R$ and where the Mach number is independent of $R$. This solution, which had previously been discovered by Spruit et al. (1987), has several interesting and unexpected properties. However, before we can explore the consequences of these properties we need first to confirm that the self-similar solution itself is real and not just an artifact of the vertical integration of the equations.

Vertical integration is a standard approximation which has been used in accretion disk studies from the earliest days. The physical motivation behind this approximation is that the vertical thickness of an accretion disk is usually much smaller than the local radius, so that the flow velocities are likely to be more or less independent of height. It is then reasonable to expect that very little is lost by integrating out the vertical coordinate. Unfortunately, the self-similar solutions discovered by Spruit et al. (1987) and NY are not thin. The temperature of the accreting gas is nearly always close to virial, and the formal vertical thickness is comparable to the radius. This inconsistency raises serious questions concerning the validity of the solutions and the reliability of the conclusions.

In this paper, we avoid the height-integration approximation and instead set up exact flow equations for steady axisymmetric flow in the $r \theta$ plane. The equations we obtain are similar to those written down by Begelman \& Meier (1982). We present here numerical self-similar solutions of the equations. These solutions are direct generalizations of the height-integrated solutions in NY. Rather gratifyingly, we find that all the features of the height-integrated solution are reproduced well in the present solutions. In fact, we even find excellent quantitative agreement between the two approaches, suggesting that the height-integration approximation may be much better than previously thought.

In $\S 2$ we write down the equations that we solve along with the boundary conditions. Then in $\S 3$ we describe numerical results of the equations, discuss some of the more interesting properties of the solutions, and make various comparisons with the previously-obtained height-integrated solutions. Finally, in $\S 4$ we discuss the implications of the results, especially for the formation of outflows. 
Appendix A compares our solutions with those obtained by Begelman \& Meier (1982), and Appendix B discusses the relationship to Bondi (1952) accretion.

\section{Equations of a Steady Axisymetric Advection-Dominated Flow}

We define the isothermal sound speed $c_{s}$ by

$$
p=\rho c_{s}^{2},
$$

where $p$ is the pressure and $\rho$ is the density, and we assume that the gas has a fixed ratio of specific heats, $\gamma \equiv c_{p} / c_{v}$. For convenience we define

$$
\epsilon=\frac{5 / 3-\gamma}{\gamma-1} .
$$

We work in spherical polar coordinates $r \theta \phi$, and write the three components of velocity as $v_{r}, v_{\theta}$ and $v_{\phi}=\Omega r \sin \theta$, where $\Omega$ is the angular velocity. The gas accretes onto a central mass $M$ and we define the Keplerian angular velocity $\Omega_{K}(r)$ by

$$
\Omega_{K}(r)=\left(\frac{G M}{r^{3}}\right)^{1 / 2}
$$

Assuming steady state $(\partial / \partial t=0)$ and axisymmetry $(\partial / \partial \phi=0)$, the continuity equation gives

$$
\frac{1}{r^{2}} \frac{\partial}{\partial r}\left(r^{2} \rho v_{r}\right)+\frac{1}{r} \frac{\partial}{\partial \theta}\left(\rho v_{\theta}\right)=0 .
$$

Integrating this over angle we obtain the net mass accretion rate,

$$
\dot{M}=-\int 2 \pi r^{2} \sin \theta \rho v_{r} d \theta .
$$

We employ the usual $\alpha$-prescription for the viscosity, which we write in the following form for the kinematic coefficient of viscosity,

$$
\nu=\frac{\alpha c_{s}^{2}}{\Omega_{K}},
$$

where $\alpha$ is a constant. This form is equivalent to the assumption that $\nu \sim \alpha c_{s} H$ where $H \sim c_{s} / \Omega_{K}$ is the "vertical" scale height. Note that $\nu$ is a function of position, both because $\Omega_{K}$ depends on $r$ and because $c_{s}$ varies from point to point. The three components of the momentum equation give (e.g., Mihalas \& Mihalas 1984)

$$
\rho\left(v_{r} \frac{\partial v_{r}}{\partial r}-\frac{v_{\phi}^{2}}{r}\right)=-\frac{G M \rho}{r^{2}}-\frac{\partial p}{\partial r}+\frac{\partial}{\partial r}\left[2 \nu \rho \frac{\partial v_{r}}{\partial r}-\frac{2}{3} \nu \rho\left(\frac{2 v_{r}}{r}+\frac{\partial v_{r}}{\partial r}\right)\right]
$$




$$
\begin{gathered}
+\frac{1}{r} \frac{\partial}{\partial \theta}\left(\frac{\nu \rho}{r} \frac{\partial v_{r}}{\partial \theta}\right)+\frac{\nu \rho}{r}\left[4 r \frac{\partial}{\partial r}\left(\frac{v_{r}}{r}\right)+\frac{\cot \theta}{r} \frac{\partial v_{r}}{\partial \theta}\right], \\
\rho\left(-\frac{\cot \theta}{r} v_{\phi}^{2}\right)=-\frac{1}{r} \frac{\partial p}{\partial \theta}+\frac{\partial}{\partial r}\left(\frac{\nu \rho}{r} \frac{\partial v_{r}}{\partial \theta}\right) \\
+\frac{1}{r} \frac{\partial}{\partial \theta}\left[\frac{2 \nu \rho v_{r}}{r}-\frac{2 \nu \rho}{3 r}\left(\frac{2 v_{r}}{r}+\frac{\partial v_{r}}{\partial r}\right)\right]+\frac{3 \nu \rho}{r^{2}} \frac{\partial v_{r}}{\partial \theta} \\
\rho\left(v_{r} \frac{\partial v_{\phi}}{\partial r}+\frac{v_{\phi} v_{r}}{r}\right)=\frac{\partial}{\partial r}\left[\nu \rho r \frac{\partial}{\partial r}\left(\frac{v_{\phi}}{r}\right)\right]+\frac{1}{r} \frac{\partial}{\partial \theta}\left[\frac{\nu \rho \sin \theta}{r} \frac{\partial}{\partial \theta}\left(\frac{v_{\phi}}{\sin \theta}\right)\right] \\
+\frac{\nu \rho}{r}\left[3 r \frac{\partial}{\partial r}\left(\frac{v_{\phi}}{r}\right)+\frac{2 \cot \theta \sin \theta}{r} \frac{\partial}{\partial \theta}\left(\frac{v_{\phi}}{\sin \theta}\right)\right]
\end{gathered}
$$

while the energy equation gives

$$
\begin{aligned}
& \rho\left(v_{r} \frac{\partial e}{\partial r}-\frac{p}{\rho^{2}} v_{r} \frac{\partial \rho}{\partial r}\right)=-\frac{2 f \nu \rho}{3}\left[\frac{1}{r^{2}} \frac{\partial}{\partial r}\left(r^{2} v_{r}\right)\right]^{2}+2 f \nu \rho \times \\
& {\left[\left(\frac{\partial v_{r}}{\partial r}\right)^{2}+2\left(\frac{v_{r}}{r}\right)^{2}+\frac{1}{2}\left(\frac{1}{r} \frac{\partial v_{r}}{\partial \theta}\right)^{2}+\frac{1}{2}\left(r \frac{\partial}{\partial r}\left(\frac{v_{r}}{r}\right)\right)^{2}+\frac{1}{2}\left(\frac{\sin \theta}{r} \frac{\partial}{\partial \theta}\left(\frac{v_{\phi}}{\sin \theta}\right)\right)^{2}\right]}
\end{aligned}
$$

Anticipating the self-similarity form assumed below (eqs 2.11-2.15), we have set $v_{\theta}=0$ in the above equations. The left-hand side of equation (2.10) is the gradient of the entropy. The right-hand side is the rate of generation of energy through viscous dissipation, except that it is multiplied by a parameter $f$. This parameter describes the fraction of the disipated energy which is advected as stored entropy, and is therefore a measure of the degree to which the flow is advection-dominated. A fraction $(1-f)$ of the energy is removed through radiative losses. In principle, $f$ could be a function of $\theta$, but all the results we present here correspond to the simplest assumption, viz. $f=$ constant.

We restrict ourselves in this paper to self-similar flows. We therefore seek a solution of the form

$$
\begin{aligned}
\rho & =r^{-3 / 2} \rho(\theta), \\
v_{r} & =\sqrt{\frac{G M}{r}} v(\theta)=r \Omega_{K}(r) v(\theta), \\
v_{\theta} & =0 \\
v_{\phi} & =r \Omega_{K}(r) \Omega(\theta), \\
c_{s} & =r \Omega_{K}(r) c_{s}(\theta) .
\end{aligned}
$$


The form of the solution is obvious. The only lengthscale in the problem is $r$ and the only frequency is $\Omega_{K}$. Therefore, all velocities must scale with radius as $r \Omega_{K}$. Angular variations at a given radius are modeled through the dimensionless functions $v(\theta), \Omega(\theta)$ and $c_{s}(\theta)$. Given the radial scaling of $v_{r}$, the scaling of $\rho$ is uniquely determined by the constancy of $\dot{M}$ in (2.5), and since $r^{2} \rho v_{r}$ is independent of $r$, the continuity equation (2.4) shows that $v_{\theta}=0$.

By construction, the solution (2.11) - (2.15) automatically satisfies the continuity eq. (2.4). Substituting the solution in the momentum and energy equations we obtain the following four coupled differential equations in $\theta$ :

$$
\begin{aligned}
-\frac{1}{2} v^{2}-\sin ^{2} \theta \Omega^{2} & =-1+c_{s}^{2}\left(\frac{5}{2}-\alpha v+\alpha \cot \theta \frac{d v}{d \theta}\right)+\frac{1}{\rho} \frac{d}{d \theta}\left(\alpha \rho c_{s}^{2} \frac{d v}{d \theta}\right) \\
-\cos \theta \sin \theta \Omega^{2} & =-\frac{1}{\rho} \frac{d}{d \theta}\left(\rho c_{s}^{2}\right)+\frac{\alpha c_{s}^{2}}{2} \frac{d v}{d \theta}+\frac{1}{\rho} \frac{d}{d \theta}\left(\alpha c_{s}^{2} \rho v\right) \\
\frac{1}{2} \sin \theta v \Omega & =-\frac{3 \alpha \sin \theta c_{s}^{2} \Omega}{4}+\frac{1}{\rho} \frac{d}{d \theta}\left(\alpha \sin \theta \rho c_{s}^{2} \frac{d \Omega}{d \theta}\right)+2 \alpha \cos \theta c_{s}^{2} \frac{d \Omega}{d \theta} 2 . \\
-\frac{3 \epsilon^{\prime} v}{2 \alpha} & =3 v^{2}+\frac{9}{4} \sin ^{2} \theta \Omega^{2}+\sin ^{2} \theta\left(\frac{d \Omega}{d \theta}\right)^{2}+\left(\frac{d v}{d \theta}\right)^{2}
\end{aligned}
$$

Following NY, we have introduced in eq. (2.19) a quantity $\epsilon^{\prime}$ which we define by

$$
\epsilon^{\prime}=\frac{\epsilon}{f}=\frac{1}{f}\left(\frac{5 / 3-\gamma}{\gamma-1}\right),
$$

where $f$ is the parameter we have already introduced in eq. (2.10).

Equations (2.16) - (2.20) constitute a sixth-order system of ordinary differential equations for the four functions $v(\theta), \Omega(\theta), c_{s}(\theta)$, and $\rho(\theta)$. The integral (2.5) sets the normalization of $\rho(\theta)$ and provides one boundary condition. The remaining boundary conditions are distributed between the equatorial plane, $\theta=\pi / 2$, and the rotation axis, $\theta=0$. At $\theta=\pi / 2$, we have by symmetry the conditions

$$
\theta=\frac{\pi}{2}: \quad \frac{d v}{d \theta}=\frac{d \Omega}{d \theta}=\frac{d c_{s}}{d \theta}=\frac{d \rho}{d \theta}=0 .
$$

At $\theta=0$, we insist that the solutions be well-behaved and non-singular. This leads to the conditions

$$
\theta=0: \quad \frac{d \Omega}{d \theta}=\frac{d v}{d \theta}=\frac{d c_{s}}{d \theta}=\frac{d \rho}{d \theta}=0, v=0 .
$$

The last condition follows from eq. (2.19). Not all of the conditions (2.21), (2.22) are independent. We choose a convenient subset of these conditions and solve the differential equations (2.16)-(2.19) using a numerical relaxation technique (e.g., Press et al. 1992).

Under certain conditions, the sixth-order set of equations (2.16)-(2.19) reduces to a second-order system. We discuss this simplification in Appendix A 
and compare our work to a previous analysis of this problem by Begelman \& Meier (1982)

Technically, equation (2.19) allows two possible boundary conditions on $v$ at $\theta=0$, viz. $v=0$ which is the one we have given in eq (2.22) and $v=-\epsilon^{\prime} / 2 \alpha$. We have tried to obtain rotating solutions which satisfy the second condition and have been unable to find any. However, the boundary condition $v=-\epsilon^{\prime} / 2 \alpha$ does allow a purely spherical non-rotating inflow solution which we discuss in Appendix B. This solution is a generalization of Bondi flow to the case when there is viscosity.

\section{Results}

\subsection{Typical Solutions}

We have obtained numerical solutions of eqs. (2.16) - (2.19) for a variety of values of the viscosity parameter $\alpha$ and the thermodynamic parameter $\epsilon^{\prime}$ (defined in eqs. (2.6) and (2.20)). Figure 1 shows a typical sequence of solutions corresponding to $\alpha=0.1$ and $\epsilon^{\prime}=0.1,1,10$. These solutions may be considered either as a sequence of fully advection-dominated flows $(f=1)$ with $\gamma=1.6061,1.3333,1.0606$, or as flows with a fixed value of $\gamma$ and with a sequence of decreasing $f$ or increasing cooling.

The four panels in Fig. 1 show the variation with polar angle $\theta$ of various dynamical quantities in the solutions. The top left panel displays the dimensionless angular velocity $\Omega(\theta)$. Rather surprisingly, we find that $\Omega$ is nearly independent of $\theta$ in each solution, varying by only $\sim 10 \%$ from $\theta=0$ to $\theta=\pi / 2$. Radial shells therefore rotate more-or-less rigidly, but of course there is differential rotation between neighboring shells. The actual value of $\Omega$ varies significantly from one solution to another, changing from $\Omega \sim 0.2$ at $\epsilon^{\prime}=0.1$ to $\Omega \sim 0.9$ at $\epsilon^{\prime}=10$. The scaling of $\Omega$ with $\epsilon^{\prime}$ follows eq (3.2). Note that $\Omega \propto\left(\epsilon^{\prime}\right)^{1 / 2}$ for $\epsilon^{\prime} \ll 1$. This implies that $\Omega \rightarrow 0$ as $\gamma \rightarrow 5 / 3$.

The top right panel shows the radial velocity profiles $v(\theta)$ of the solutions. The velocity is zero at $\theta=0$ (this is a boundary condition) and maximum at $\theta=\pi / 2$. We find that $v$ is essentially independent of $\epsilon^{\prime}$ for $\epsilon^{\prime} \ll 1$ and varies as $v \propto 1 / \sqrt{\epsilon^{\prime}}$ for $\epsilon^{\prime} \gg 1$ (see eq 3.1).

The bottom left panel shows profiles of the density $\rho(\theta)$. In the $\epsilon^{\prime}=0.1$ solution $\rho$ varies by only $\sim 10 \%$ from $\theta=0$ to $\theta=\pi / 2$. The solution therefore corresponds to a nearly spherical configuration. This is demonstrated in Figure 2 (top left) where we display isodensity contours in the meridional plane. The resemblance of this solution to a star is striking, but of course it is not a normal star, since it involves a steady accretion flow. In any case, it is quite clear that this solution is very definitely not a "disk" in the usual sense. The density contrast between $\rho(0)$ and $\rho(\pi / 2)$ increases with increasing $\epsilon^{\prime}$, becoming a factor $\sim 2$ at $\epsilon^{\prime}=1$, and a factor $\sim 50$ at $\epsilon^{\prime}=10$. The isodensity contours of these solutions are shown in the top right and bottom left panels of Fig. 2. The $\epsilon^{\prime}=1$ solution looks like a rotationally flattened star, while the $\epsilon^{\prime}=10$ solution is beginning to resemble a standard thin disk. A value of $\epsilon^{\prime}=10$ normally implies a small value 
of $f$ (unless $\gamma$ is close to 1 ), and so the solution represents a case where there is significant cooling. This is precisely the limit where we expect the flow to occur in a thin disk.

The $v(\theta)$ and $\rho(\theta)$ profiles both peak at $\theta=\pi / 2$. Therefore, in all our solutions the bulk of the accretion occurs along the equatorial plane, and the accretion rate goes to zero along the rotation pole.

The bottom right panel of Fig. 1 shows the variation of $c_{s}^{2}$, or equivalently the gas temperature, with $\theta$. In the $\epsilon^{\prime}=0.1$ solution, $c_{s}^{2}$ is almost independent of $\theta$, and the pressure $p=\rho c_{s}^{2}$ too is independent of $\theta$. In this solution, the rotation is highly sub-Keplerian and so hydrostatic equilibrium requires primarily a balance between gravity and the pressue gradient. Since gravity acts in the radial direction, the pressure gradient too is almost radial. The $\epsilon^{\prime}=1$ and especially the $\epsilon^{\prime}=10$ solutions have larger temperature and pressure variations with $\theta$ and have non-radial pressure gradients. This is to be expected given the more rapid rotation of these solutions and the increasing importance of centrifugal acceleration.

An interesting feature of the large $\epsilon^{\prime}$ solutions is worth emphasizing. As already mentioned, these solutions have efficient cooling and therefore resemble thin disks. Nevertheless, in all cases there is a low density corona above the disk which is at nearly virial temperature. This is illustrated by the $\epsilon^{\prime}=10$ solution in Fig. 1. The hottest temperature is achieved at the rotation poles, $\theta=0, \pi$.

In addition to the above examples with $\alpha=0.1$, we have calculated a number of solutions with other values of $\alpha$. For $\alpha \ll 1, v$ scales as $\alpha$, but except for this, solutions with the same $\epsilon^{\prime}$ but with different values of $\alpha$ are virtually indistinguishable from one another. There are more significant variations when $\alpha$ exceeds unity. However, such large values of $\alpha$ are probably unlikely (e.g. Narayan, Loeb \& Kumar 1994, Hawley, Gammie \& Balbus 1994), and we have not explored this region of parameter space.

In the advection-dominated limit, where $\epsilon^{\prime} \ll 1$, our solutions have very simple scalings, viz. $v \sim-\alpha, \Omega \sim\left(\epsilon^{\prime}\right)^{1 / 2}, c_{s} \sim 1$. These scalings arise as follows. Since the cooling is inefficient, all the viscous energy is stored in the accreting gas, and this means that the thermal velocities approach virial speeds, i.e $c_{s} \sim 1$. Comparing the various terms in eq $(2.18)$ we see that $v$ has to scale as $-\alpha c_{s}^{2}$. This gives $v \sim-\alpha$ for a virial gas. The physical reason for the scaling is that the radial velocity in an accretion flow is determined primarily by the rate at which angular momentum is removed from the gas and this depends on the viscosity coefficient $\nu$. In the $\alpha$ prescription (eq 2.6), we have $\nu \sim \alpha$ in scaled units and this therefore implies $v \sim-\alpha$. Finally, the scaling of $\Omega$ arises through the energy equation (2.19). The left-hand side of this equation is the product of the radial gradient of the entropy and the radial velocity, and therefore represents the steady state rate of change of entropy of a parcel of accreting gas. Recall that our solutions are by construction self-similar with $\rho \propto r^{-3 / 2}$ and $p \propto r^{-5 / 2}$. If $\gamma$ is exactly equal to $5 / 3$, then a flow with these radial dependences is automatically isentropic. However, for $\gamma \neq 5 / 3$, there is an entropy gradient in the flow such that the entropy increases inwards whenever $\gamma<5 / 3$, i.e. $\epsilon^{\prime}>0$. This means that the entropy of each accreting gas element increases with time at a rate proportional to $\epsilon^{\prime}$. The entropy has to be generated of course by the viscous energy dissipation, described by the four terms in the right of eq (2.19). Usually, the dissipation is 
dominated by the two terms proportional to $\Omega^{2}$ and $(d \Omega / d \theta)^{2}$ which arise from the $r \phi$ and $\phi z$ components of the shear stress. Thus the dissipation is proportional to $\Omega^{2}$. In order to achieve self-consistency, the magnitude of $\Omega$ in the self-similar solution is adjusted such that the dissipated energy exactly matches the energy that is required to maintain the self-similar entropy gradient. Since the latter is proportional to $\epsilon^{\prime}$ and since $v \sim-\alpha$, we thus see from (2.19) that we require $\Omega \sim\left(\epsilon^{\prime}\right)^{1 / 2}$. This is exactly what we see in our numerical solutions.

A somewhat interesting feature of eq (2.19) is that even when $\Omega \rightarrow 0$, the right hand side still remains non-zero. This is because of the terms $v^{2}$ and $(d v / d \theta)^{2}$, the first of which is the viscous dissipation arising purely from the geometric convergence of the flow due to the spherical geometry, while the second represents the $r z$ component of the shear stress. These two terms imply a certain minimum level of viscous dissipation even in a very slowly rotating flow. This dissipation will of course cause the entropy to increase inwards, and by the arguments given above we see that $\epsilon^{\prime}$ has to be greater than a certain minimum value. Indeed, we have discovered from our numerical experiments that self-similar solutions exist only for $\epsilon^{\prime} \gtrsim C \alpha^{2}$, where $C$ is a constant of order unity. The limit is exactly of the form we expect from eq (2.19). Thus for large values of $\alpha$, the parameter $\gamma$ needs to deviate significantly from $5 / 3$ and/or $f$ needs to be quite different from unity in order to have a self-similar flow. On a related point, we note that there is a second branch of solutions which corresponds to non-rotating purely spherical accretion. These solutions are quite distinct from the $\Omega \rightarrow 0$ limit of the rotating solutions discussed here and are in fact closely related to Bondi (1952) spherical accretion. We discuss these solutions in Appendix B.

In addition to the solutions described so far, we have found (for somewhat large values of $\epsilon^{\prime} / \alpha^{2}$ ) other solutions where $\Omega$ reverses sign one or more times as a function of $\theta$. These higher-order solutions come in two parities. Solutions with even parity have an even number of nodes in $\Omega(\theta)$ between $\theta=0$ and $\theta=\pi$. These solutions satisfy the boundary conditions $(2.22)$ at the equator, and have rotation profiles which are symmetric between the two hemispheres. The lower right panel of Fig. 2 shows isodensity contours of one such solution with two nodes where the flow down the two poles rotates in one sense while the flow in the equatorial plane rotates with the opposite sense. Solutions with odd parity have an odd number of nodes, and the rotation profiles in the two hemispheres are reversed with respect to each other. These solutions satisfy the boundary condition $\Omega=0$ at $\theta=\pi / 2$. We do not expect any of these higherorder solutions to be relevant except in rare cases where the initially infalling gas happens to have reversals in the sign of the angular momentum as a function of $\theta$. In the rest of the paper we restrict ourselves to the nodeless fundamental solutions.

The results described so far correspond to the particular form of viscosity given in eq. (2.6). To check how sensitive the results are to the viscosity prescription, we obtained solutions corresponding to a second law. By dimensional analysis we see that self-similarity is possible only if $\nu$ scales with radius as $r^{1 / 2}$. Therefore, as our alternate prescription we used $\nu=\alpha c_{s} r$. We found that the solutions with this viscosity law are very similar to those described above. Therefore, none of our results are special to the particular viscosity prescription we have adopted. 


\subsection{Comparison with Height-Integrated Solutions}

One of the primary aims of this study was to check the validity of the height-integrated approximation for advection-dominated flows. As we have seen, accretion flows become very nearly spherical when they are advection-dominated $(f \rightarrow 1)$. From this it might appear that the height-integrated approximation, which is based on a disk-like picture of the flow, would be particularly inappropriate in this limit. We investigate the issue quantitatively.

Spruit et al. (1987) and NY showed that the height-integrated equations have the following analytical self-similar solution,

$$
\begin{aligned}
(v)_{h} & =-\left(5+2 \epsilon^{\prime}\right) \frac{g\left(\alpha, \epsilon^{\prime}\right)}{3 \alpha} \approx-\frac{3 \alpha}{\left(5+2 \epsilon^{\prime}\right)} \\
(\Omega)_{h} & =\left[\frac{2 \epsilon^{\prime}\left(5+2 \epsilon^{\prime}\right) g\left(\alpha, \epsilon^{\prime}\right)}{9 \alpha^{2}}\right]^{1 / 2} \approx\left[\frac{2 \epsilon^{\prime}}{5+2 \epsilon^{\prime}}\right]^{1 / 2}, \\
\left(c_{s}^{2}\right)_{h} & =\frac{2\left(5+2 \epsilon^{\prime}\right)}{9} \frac{g\left(\alpha, \epsilon^{\prime}\right)}{\alpha^{2}} \approx \frac{2}{5+2 \epsilon^{\prime}}
\end{aligned}
$$

where the second relation in each equation refers to the limit $\alpha \ll 1$, and the subscript $h$ is to remind us that these expressions correspond to the heightintegrated approximation. The function $g\left(\alpha, \epsilon^{\prime}\right)$ is given by

$$
g\left(\alpha, \epsilon^{\prime}\right) \equiv\left[1+\frac{18 \alpha^{2}}{\left(5+2 \epsilon^{\prime}\right)^{2}}\right]^{1 / 2}-1
$$

We now take the exact solutions calculated in this paper and extract from them three fiducial values each of $v, \Omega$ and $c_{s}^{2}$. First, we consider the values of the variables at the mid-plane, $\theta=\pi / 2$; we refer to these values as $(v)_{m},(\Omega)_{m}$ and $\left(c_{s}^{2}\right)_{m}$. As our second estimate, we compute spherically-averaged values, e.g.

$$
\langle v\rangle_{\theta}=\frac{\int_{0}^{\pi / 2} v(\theta) \rho(\theta) d \theta}{\int_{0}^{\pi / 2} \rho(\theta) d \theta}
$$

with $\langle\Omega\rangle_{\theta}$ and $\left\langle c_{s}^{2}\right\rangle_{\theta}$ defined similarly. Finally, we compute z-averaged values, which correspond to cylindrical averages parallel to the rotation axis. In this case, we define the averages according to

$$
\begin{gathered}
\langle v\rangle_{z}=\frac{\int_{0}^{\infty} v(z) \sin (\theta) \rho(z) d z}{\int_{0}^{\infty} \rho(z) d z}, \\
\langle\Omega\rangle_{z}=\frac{\int_{0}^{\infty} \Omega(z) \rho(z) d z}{\int_{0}^{\infty} \rho(z) d z}
\end{gathered}
$$




$$
\left\langle c_{s}^{2}\right\rangle_{z}=\frac{\int_{0}^{\infty} c_{s}^{2}(z) \rho(z) d z}{\int_{0}^{\infty} \rho(z) d z} .
$$

Figure 3 compares these three fiducial values of $v, \Omega$ and $c_{s}^{2}$ from the exact solutions with the corresponding height-integrated values, $(v)_{h},(\Omega)_{h},\left(c_{s}^{2}\right)_{h}$, for a range of $\epsilon^{\prime}$ extending from $\epsilon^{\prime}=10^{-2}$ to $10^{2}$. The results correspond to small values of $\alpha \lesssim 0.1$; in this regime, $\Omega$ and $c_{s}^{2}$ are independent of $\alpha$ while $v$ is simply proportional to $\alpha$.

When $\epsilon^{\prime} \gg 1$, we are in the cooling-dominated regime where the flow resembles standard thin disk accretion. In this limit, we expect height-integration to be quite accurate, and indeed we do find that the height-integrated estimates $(v)_{h}$, $(\Omega)_{h},\left(c_{s}^{2}\right)_{h}$ agree well with all three estimates obtained from the exact numerical solutions. The only discrepancy is between $\left(c_{s}^{2}\right)_{h}$ and $\left(c_{s}^{2}\right)_{m}$, but this can be understood. These solutions have coronae as discussed in $\S 3.1$, and naturally the midplane value of $c_{s}^{2}$ is smaller than either the spherical average or the $z$-average.

When $\epsilon^{\prime}$ falls below unity the flow becomes quite spherical, and we would expect height-integration to be less valid. As expected, we find that the $z$-averaged values of $v, \Omega$ and $c_{s}^{2}$ differ significantly from the height-integrated estimates. For instance, at $\epsilon^{\prime}=10^{-2}$, we find $\langle v\rangle_{z}=0.32(v)_{h},\langle\Omega\rangle_{z}=0.42(\Omega)_{h},\left\langle c_{s}^{2}\right\rangle_{z}=$ $0.46\left(c_{s}^{2}\right)_{h}$. Thus, height-integration leads to fairly large errors at the level of factors $\sim 2-3$.

However, Fig. 3 reveals a surprise, viz. that the height-integrated estimates agree very well with both the midplane values and the spherically-averaged values of the exact solutions. For instance, at $\epsilon^{\prime}=10^{-2}$, we find $(v)_{m}=1.28(v)_{h}$, $(\Omega)_{m}=1.13(\Omega)_{h},\left(c_{s}^{2}\right)_{m}=1.00\left(c_{s}^{2}\right)_{h}$ for the midplane values, and $\langle v\rangle_{\theta}=0.82(v)_{h}$, $\langle\Omega\rangle_{\theta}=1.09(\Omega)_{h},\left\langle c_{s}^{2}\right\rangle_{\theta}=1.00\left(c_{s}^{2}\right)_{h}$ for the spherical averages. The spherical averages show particularly good agreement with the height-integrated estimates over the entire range of $\epsilon^{\prime}$ from 0 to infinity, with no error exceeding $20 \%$.

This result suggests that one should interpret the height-integrated equations, not as averages over cylindrical height $z$, but rather as averages over spherical polar angle $\theta$ at a fixed $r$. Once this is done, height-integration is a good approximation even for nearly spherical flows. The height-integrated equations, especially in the form of the so-called "slim disk" equations (Abramowicz et al. 1988), have become popular in recent years for modeling the dynamics of accretion flows. Until now, it has not been clear exactly how slim a disk has to be in order for the equations to be valid, and also exactly what kind of an average the solutions represent. Based on the results presented here, we suggest that the slim disk equations may be applied virtually to any accretion flow around a point mass, however non-slim the flow may be, and that the results should be interpreted as spherical averages rather than as $z$-averages. We caution, however, that this suggestion is based on the properties of a very special class of self-similar solutions, and needs to be tested on non-self-similar flows.

\subsection{The Bernoulli Parameter}

The Bernoulli parameter $B e$, defined as the sum of the kinetic energy, the potential energy and the enthalpy of the accreting gas, is of interest in accretion flows because it measures the likelihood that outflows or winds may originate 
spontaneously (NY). An adiabatic flow has a constant $B e$ along streamlines. If $B e$ is positive for any of the accreting gas, then this gas can potentially reach infinity with a net positive kinetic energy.

Let us normalize $B e$ in our self-similar solutions by the square of the local free-fall speed, $v_{f f}=\Omega_{K} r$, and consider the dimensionless parameter

$$
b \equiv \frac{B e}{\Omega_{K}^{2} r^{2}}=\frac{1}{2} v^{2}+\frac{1}{2}(\Omega \sin \theta)^{2}-1+\frac{\gamma}{\gamma-1} c_{s}^{2} .
$$

The parameter $b$ is a function of $\theta$ but independent of $r$. In NY we showed that the Bernoulli parameter is positive in height-integrated advection-dominated flows, and suggested that this may explain the frequent occurrence of outflows and winds in many accretion systems. However, because the result was obtained through the height-integrated equations, it had to be treated with caution. We now consider the behavior of $b$ in the exact self-similar solutions obtained in this paper.

Fig. 4 shows $b(\theta)$ for a sequence of solutions. All the solutions have the same $\epsilon=0.333(\gamma=1.5)$, but the advection-domination parameter $f$ varies over the range $f=1,0.446,0.33,0.033,0.0033,0.0011$, which means that $\epsilon^{\prime}=$ $0.33,0.75,1,10,100,300$. We confirm the basic result of NY that advectiondominated flows have positive $b$. Furthermore, we see that for $f>0.446, b(\theta)>0$ at all $\theta$. This means that for flows that are so highly advection-dominated, the entire gas has positive Bernoulli parameter. Even in the limit of extreme cooling $(f \rightarrow 0)$, there is always some gas near the rotation axis $(\theta \rightarrow 0)$ which has a positive $b$. The reason for this is the corona which we mentioned in $\S 3.1$. Even though the disk cools efficiently and is geometrically thin, it still has a hot virial corona above it and this region of the flow can acquire a positive $b$ especially close to the rotation axis.

These results make the connection between accretion and outflows much stronger than we suspected based only on the height-integrated work of NY. A case can be made now that perhaps all accretion flows, whether advectiondominated or cooling-dominated, are capable of producing outflows. The main difference between the two kinds of flow may be only a quantitative one, viz. the former probably produce more powerful outflows than the latter. Also, it would appear that outflows will prefer to form along the rotation axis, since this is where $b$ is most positive in all cases. A bipolar morphology is thus natural. The argument is made even stronger by the results on convection discussed in the next section.

It is important to emphasize that the positivity of $b$ does not imply a violation of energy conservation. In viscous accretion flows, the energy content of a parcel of gas is modified by viscous transport of energy from one radius to another and from one $\theta$ to another. Let us consider only the radial flux for simplicity. The radial flux of energy transported by viscosity is equal to the quantity (the radial angular momentum flux) $\times$ (the angular velocity) $\sim \dot{M} v_{\phi}^{2}$. This energy flux is directed outward and has a negative divergence. Therefore, it deposits energy at each radius. For a given $\dot{M}$, the rate of deposition of energy is maximum in the case of a standard thin accretion disk, where $v_{\phi}=\Omega_{K} r$. However, because these 
disks also cool efficiently, the deposited energy does not produce an enhancement of $b$ (in fact, $b=-0.5$ ), but rather leads to an enhancement of the locally radiated flux. This explains the well-known result that the radiative flux emerging from a thin disk (except near the inner edge) is three times larger than the rate at which gravitational energy is released locally (e.g. Frank, King \& Raine 1989). As the parameter $f$ increases and the flow becomes more advection-dominated, the viscous energy flux actually decreases in magnitude because $v_{\phi}$ becomes smaller than $\Omega_{K} r$. However, because the cooling is also less efficient, a larger fraction of the energy is retained by the gas and therefore, paradoxically, $b$ actually becomes larger. As we have seen, when $f$ exceeds a critical value, $b$ can actually become positive over the entire flow.

The $\theta$ component of the viscous stress causes angular redistribution of the energy at a given $r$. The direction of this flux is such as to enhance $b$ at the poles relative to the equator. The result is that the pole always has a positive $b$ even in the limit of small $f$.

The self-similar solution is very special in that it has an infinite source of energy at $r=0$ which can be transported outward by viscosity. This explains how the entire solution can have a positive $b$. If we consider a non-infinite flow which is terminated at a finite inner radius $r_{i}$, then for radii close to $r_{i}$ the viscous energy flux will be less than the self-similar value. The gas near the inner edge will therefore have negative $b$. However, once we are reasonably far from the inner edge (say $r>$ few $\times r_{i}$ ), the self-similar value of $b$ will be achieved and beyond this radius the flow will be indistinguishable from the self-similar form. Overall, the deficit of $b$ near the inner edge will compensate for the positive $b$ elsewhere, ensuring that energy is conserved globally.

\subsection{Convection}

In NY, we showed that advection-dominated flows have entropy increasing inwards and therefore that these flows are intrinsically unstable to convective instabilities. This point was made earlier by Begelman \& Meier (1982). We discuss here the role of convection in the solutions described in this paper.

The left-hand side of eq (2.19) is proportional to $v T d s / d r$, where $d s / d r$ is the radial derivative of the entropy. Since $v$ is negative and the right-hand side of eq (2.19) is positive (it consists only of dissipation terms), we see that $d s / d r$ is negative at all $\theta$, and therefore that the flow is convectively unstable at all angles. In any simple theory of convection we expect the convective flux to be parallel to the local pressure gradient since this is the direction associated with the buoyancy force. (In principle, the flux could be in a different direction in a rotating flow because of anisotropic transport, but we ignore this complication.) We have seen earlier that whenever the flow is advection-dominated $\left(f \rightarrow 1, \epsilon^{\prime} \lesssim 1\right)$ the flow is nearly spherical and the pressure gradient is almost radial. Therefore, we expect convection to act primarily in the radial direction. This is very different from the vertical convection which is usually discussed in the context of thin accretion disks (e.g. Ryu \& Goodman 1992). 
When there is convection we can expect it to have a back reaction on the basic flow. To estimate the magnitude of the effect, let us rewrite the energy equation (2.19) with the convective contribution included:

$$
-\frac{3 \epsilon}{2} \frac{\rho v_{r} c_{s}^{2}}{r}=-\nabla \cdot \mathbf{F}_{\mathbf{c}}+\frac{f \alpha \rho c_{s}^{2}}{\Omega_{K}}\left[\frac{3 v_{r}^{2}}{r^{2}}+\frac{1}{r^{2}}\left(\frac{d v_{r}}{d \theta}\right)^{2}+\frac{9}{4} \sin ^{2} \theta \Omega^{2}+\sin ^{2} \theta\left(\frac{d \Omega}{d \theta}\right)^{2}\right] .
$$

Here $\mathbf{F}_{\mathbf{c}}$ is the convective energy flux, and $\rho, v_{r}, \Omega, c_{s}$ refer to the physical variables and not the scaled functions defined in eqs $(2.11)-(2.15)$. The term on the left of eq (3.10) is the divergence of the advected entropy flux, $\rho T \mathbf{v} \cdot \nabla s$, which we refer to as the "advection term." The first term on the right is the net deposition of energy due to the inflow of convective energy flux into the gas the "convection term" - and the second term is the energy deposition due to viscous dissipation (reduced by our usual factor $f$ ).

To estimate the magnitude of the convection term, we note that we expect the convective flux to be proportional to the entropy gradient with some effective diffusion constant $K_{c}$. Further, we may write $K_{c}$ approximately in a form similar to the $\alpha$-prescription for viscosity. Thus, we have

$$
\mathbf{F}_{\mathbf{c}} \approx-K_{c} \rho T(\nabla s \cdot \hat{r}) \hat{r} \approx-\frac{\alpha_{c} c_{s}^{2}}{\Omega_{K}} \rho T(\nabla s \cdot \hat{r}) \hat{r}
$$

where we have assumed that $\mathbf{F}_{\mathbf{c}}$ is parallel to the unit radial vecotr $\hat{r}$ because the pressure gradient is primarily radial. Since $\nabla s \cdot \hat{r}$ is negative we see that the convective flux flows outwards. In an advection-dominated self-similar flow $c_{s}^{2} \lesssim 2 \Omega_{K}^{2} r^{2} / 5$ (see eq 3.3), and $F_{c}$ is proportional to $r^{-3}$. Therefore, the convection term becomes

$$
-\nabla \cdot \mathbf{F}_{\mathbf{c}}=\frac{F_{c}}{r} \lesssim \frac{3}{5} \alpha_{c} \epsilon \rho c_{s}^{2} \Omega_{K}
$$

We see that this term has the same form as the advection term on the left of equation (3.10). We can therefore compare the two terms directly. Figure 5 shows the ratio of the convection and advection terms for one of our advection-dominated solutions, assuming $\alpha_{c}=\alpha / 2$. The shape of the curve may be understood as follows. For small $\epsilon^{\prime}$, the radial velocity $v$ is given quite accurately by

$$
v(\theta) \approx-\frac{3}{4} \alpha \sin ^{2} \theta \Omega_{K} r
$$

Therefore, we expect

$$
\frac{\text { Convection }}{\text { Advection }} \approx \frac{8}{15 \sin ^{2} \theta} \frac{\alpha_{c}}{\alpha} .
$$

The ratio is smallest in the equatorial plane $(\theta \rightarrow \pi / 2)$ and diverges towards the rotation axis $(\theta \rightarrow 0, \pi)$.

On general grounds, we would argue that $\alpha_{c}$ cannot exceed $\alpha$. The reason is that $\alpha$, which describes the viscous transport of angular momentum, is expected 
to have contributions from many sources of viscosity such as magnetic stresses, convection, and other fluid instabilities. Convective energy transport on the other hand depends only on the strength of the convection. We therefore expect $0 \leq$ $\alpha_{c} \leq \alpha$, with the upper equality being achieved only in the limit of massively efficient convection with no source of viscous stress other than convection. In fact, if magnetic stresses always saturate at equipartition (e.g. Gammie, Hawley $\&$ Balbus 1994), then the inequality becomes $0 \leq \alpha_{c} \leq \alpha / 2$.

Figure 5 and eq (3.15) show that for a wide range of $\theta$, the convection term is smaller than the advection term, so that convection has only a moderate effect on the flow. To see this in more detail, we take the convection term to the left-hand side of eq (3.10) and combine it with the advection term (NY). This gives

$$
\text { Advection }- \text { Convection }=\frac{3 \epsilon}{2} \frac{\rho v c_{s}^{2}}{r}\left(1-\frac{8}{15 \sin ^{2} \theta} \frac{\alpha_{c}}{\alpha}\right) .
$$

With this modification, eq (2.19) continues to be valid except that we have to rewrite $\epsilon^{\prime}$ as

$$
\epsilon^{\prime}=\frac{\epsilon}{f}\left(1-\frac{8}{15 \sin ^{2} \theta} \frac{\alpha_{c}}{\alpha}\right) .
$$

The new factor in parentheses modifies the value of $\epsilon^{\prime}$. So long as this factor is positive, it has only a minor effect on the solution. The value of $\epsilon^{\prime}$ is reduced (in an angle-dependent way because of the $\sin ^{2} \theta$ ) and the flow becomes effectively more advection-dominated. The overall structure of the flow, however, remains basically unchanged. Since the convection term is smaller than the advection term, the timescale on which convection changes the entropy profile is longer than the advection timescale, and there is just not enough time for convection to have a large effect.

There is however a critical angle $\theta_{\text {crit }}$, whose value is given by eq (3.16),

$$
\theta_{\text {crit }} \approx \sin ^{-1}\left[\left(\frac{8 \alpha_{c}}{15 \alpha}\right)^{1 / 2}\right]
$$

below which convection dominates over advection. This means that for $\theta<\theta_{\text {crit }}$ the flow $i s$ strongly modified. Indeed, at these angles, convection will overwhelm the advection and the whole self-similar flow will break down. At these angles, we expect that convection will rapidly transfer entropy outwards. Since the Bernoulli constant is positive at these angles (see §3.3) the gas at large radius will acquire a great deal of positive energy and in all likelihood will flow out supersonically.

We thus have a plausible scenario for the formation of bipolar outflows. Our qualitative picture is that the accretion occurs primarily in a thick equatorial belt with $\theta_{\text {crit }}<\theta<\pi-\theta_{\text {crit }}$. Over this zone the solution is almost of the self-similar form described in this paper, and although it is convective the convection has only a minor effect on the parameters of the flow. However, for $\theta<\theta_{\text {crit }}$ and $\theta>\pi-\theta_{\text {crit }}$, the convection becomes strong enough to transfer energy outward and to disrupt the self-similar accretion. This gas has a positive Bernoulli constant (§3.3), and therefore very likely will be driven out in an outflow. Of course, we 
still don't have a self-consistent description of the outflow region, since all we have shown is that our self-similar advection-dominated solution is violently unstable in the region around the rotation axis. Nevertheless, we feel that our picture is plausible and that some outflows at least may well be generated in this manner.

Figure 5 shows that for $\alpha_{c}=\alpha / 2$, we have $\theta_{\text {crit }} \sim 33^{\circ}$. The outflow region is therefore not very wide. Moreover, the amount of mass involved in the outflow region is quite small. Let us assume that the mass outflow rate $\dot{M}_{\text {out }}$ is equal to the mass accretion rate in the original self-similar solution for $\theta<\theta_{\text {crit }}$. While the density $\rho$ is essentially independent of $\theta$ in an advection-dominated flow, the accretion velocity varies as $v \propto \sin ^{2} \theta$ (eq 3.14) and is very small in the region around the axis. From our numerical solution we calculate that $\dot{M}_{\text {out }} \sim 0.017 \dot{M}$ for $\alpha_{c}=\alpha / 2$. Therefore, only a small fraction of the accreting mass participates in the outflow. Indeed this estimate of $\dot{M}_{\text {out }}$ is probably too high since it assumes that the original self-similar flow has been set up in the polar regions and is then turned round into an outflow. In practice we imagine that the outflow will clear out two conical regions and that the mass for the outflow will be supplied by pressure gradients in the upper layers of the equatorial inflow. The mass outflow rate will then be even smaller than our estimate.

In any case, the important point is that, in the very nature of the flow, the mass in the outflow region acquires the most positive energy (or Bernoulli constant) at the expense of the rest of the accreting material. It is therefore ejected with a speed comparable to the free fall speed at the radius from which the outflow originates. The most energetic ejected material will have a speed at infinity comparable to the virial speed at the surface of the accreting star.

\section{Summary and Discussion}

The main aim of this investigation was to obtain axisymmetric self-similar advection-dominated flow solutions in three dimensions without using the heightaveraging approximation. We have succeeded in this enterprise. The solutions we have presented are obtained by numerically solving a general sixth-order system of differential equations, where the only serious approximation we have made is the use of an isotropic $\alpha$ viscosity. To our knowledge, these solutions are one of the very few fully self-consistent, axisymmetric, rotating steady state flows known with non-trivial viscous interactions. Related previous work has been published by Begelman \& Meier (1982), Liang (1988) and Henriksen \& Valls-Gabaud (1994). However, some of these other solutions have unphysical boundaries where the assumptions break down, whereas our solutions describe fully consistent equilibrium flows which fill the entire $r \theta$ plane.

Our solutions span a two-parameter family labeled by the viscosity parameter $\alpha$ (see eq. 2.6 for the definition) and a thermodynamic parameter $\epsilon^{\prime}$ (eq. 2.20). The latter is a function of the ratio of specific heats $\gamma$ of the accreting gas and the fraction $f$ of the dissipated energy which is advected with the gas. For a given $\alpha$, solutions with large values of $\epsilon^{\prime}$ behave like standard thin disks, as might be expected since these solutions correspond to $f \rightarrow 0$ and so advect very little energy. In the opposite advection-dominated limit, which corresponds to $f \rightarrow 1$ 
or $\epsilon^{\prime}<1$, our solutions describe nearly spherical flows which rotate at much below the Keplerian rate. These advection-dominated solutions have very similar properties to the approximate solutions derived in NY.

We emphasize that the solutions we find are not the usual tori with steep funnels that are normally considered when "thick accretion disks" are discussed (e.g. Begelman \& Meier 1982, Frank et al. 1992). Our solutions are much more like slowly rotating stars, except that they are not static but involve viscouslydriven settling flows. The question now arises: do our equations permit solutions with empty funnels, as described by Begelman \& Meier (1982)? As we discuss in the Appendix, this must be considered an open question at this time.

We have compared the numerical three-dimensional solutions of this paper with the approximate two-dimensional height-integrated solutions described by Spruit et al. (1987) and NY. Because some of our solutions are nearly spherical, we might expect the height-integrated solutions to be in error by quite large factors. Instead, we find that the height-integrated solutions agree very well (errors $<20 \%$ ) with spherically-averaged quantities from the exact numerical solutions. This is very encouraging since it means that simple height-integrated equations such as the "slim disk" equations (Abramowicz et al. 1988) may be valid over a much wider range of conditions than suspected before. The result also clarifies how to interpret the height-integrated equations. One should consider these equations to represent the properties of spherically-averaged quantities in the accretion flow (averages over spherical polar angle $\theta$ at fixed spherical radius $r$ ) rather than of vertically averaged quantities (averages over vertical height $z$ at fixed cylindrical radius $R$ ). This interpretation is, however, based on a very special class of solutions and should be checked on less special flows.

Advection-dominated accretion flows have a very unique feature in that they are characterized by positive values of the Bernoulli parameter $b$ (eq 3.9). This result was discovered by NY using the height-integrated equations, and is confirmed here with more exact calculations. We interpret a positive Bernoulli parameter as an indication that the accreting gas may be able spontaneously to generate winds and outflows. In some of our highly advection-dominated solutions we find that the entire accreting gas has a positive $b$ (e.g. the solutions with $f \geq 0.446$ in Fig. 4). These flows may be particularly susceptible to violent outflows. Rather surprisingly, even solutions with efficient cooling $(f \rightarrow 0)$ have hot low density coronae with positive $b$. This might mean that even regular thin accretion disks may always be accompanied by advection-dominated coronae which can drive lowdensity winds. Further, both in the advection-dominated and cooling-dominated limits, $b$ is maximally positive along the rotation axis. This suggests that whenever there is an outflow, it is likely to have a generic bipolar morphology.

Another interesting property of advection-dominated flows is that they are always convectively unstable, as was noted originally by Begelman \& Meier (1982). Because advection-dominated flows have almost spherical isobars, we argue that the convection will occur almost purely in the radial direction. This is very different from the "vertical convection" which has been studied in the context of thin accretion disks (e.g. Ryu \& Goodman 1992).

Convection in advection-dominated flows may be a source of turbulent viscosity. Although viscosity is required in order to have any accretion at all, for long it was unclear what the source of the viscosity in accretion disks may be 
since no linear hydrodynamic instability could be identified in Keplerian disks. With the recent work of Balbus \& Hawley (1991), interest therefore shifted to magnetic stresses generated through an MHD instability. The only model that we are aware of that explains accretion disk viscosity through a purely hydrodynamic model is that of Dubrulle (1992) who uses a finite amplitude instability. This model, however, results in a very small $\alpha \ll 1$. The convective instability which we identify in our advection-dominated flows is a true linear instability. Furthermore, if the flow is self-similar, then the convection also will automatically be self-similar, and this means that the convective viscosity will necessarily have a form similar to the $\alpha$-scaling given in eq. (2.6). Our models are thus rather close to being perfectly self-consistent in the sense that the viscosity is not imposed in an ad hoc manner but could, if necessary, itself be determined self-consistently from an instability in the flow.

By comparing the convective time scale with the advective time scale, we find that for most regions of our solutions, convection acts only as a moderate perturbation which changes the parameters of the flow by a modest amount but does not destroy the overall structure of the flow. However, for a range of angles around the rotation axis, $\theta<\theta_{\text {crit }} \sim 30^{\circ}$, the convection is capable of overwhelming the advection and may transport entropy outwards more rapidly than it can be carried in by accretion. Since this region of the flow is also the zone with the largest Bernoulli parameter, we suggest that the violent convection and the positive $b$ will together act to reverse the local accretion flow into a bipolar outflow. The speed of the ejected material at infinity will be of order the free-fall speed at the radius of origination of the material. We imagine that the outflow will be fed by surface material from the equatorial inflowing gas.

We feel that this scenario is a plausible and generic mechanism to produce outflows. For the mechanism to work, we require the outflowing material to remain adiabatic and to retain its Bernoulli parameter long enough to be accelerated beyond the escape velocity. Given this requirement, we suggest that an outflow can be created merely with viscous and convective redistribution of energy in the manner we have described, without any additional agencies. Hydrodynamic models of outflows have been discussed before by Eggum, Coroniti \& Katz (1988), Liang (1988) and Henriksen \& Valls-Gabaud (1994). In the former two papers, the outflow is accelerated by radiation pressure, while magnetic stresses appear to play a role in the last work. Radiation and magnetic fields doubtless help, but we suggest that outflows are possible even without them.

Note that we do not at this time have a truly self-consistent description of the outflow. By insisting on self-similarity and a constant $\dot{M}$ at all radii, we are forced to set $v_{\theta}=0$, and because of this our solutions do not have the option of diverting any of the accreting mass into an outflow. The condition of constant $\dot{M}$ is relaxed by some other authors (e.g. Liang 1988, Henriksen \& Valls-Gabaud 1994), and they do find solutions with outflows. It is not clear, however, whether their outflows are driven by the same mechanism that we suggest. It would be of interest to reformulate our problem with a non-constant $\dot{M}$ to investigate whether or not self-similar outflows are possible with these equations.

The principal characteristic of an advection-dominated accretion flow is that most of the dissipated energy is stored within the gas. This can happen at 
very low accretion rates when the gas density is so low that cooling through bremsstrahlung is inhibited. Such an effect was seen in detailed numerical models of boundary layers in cataclysmic variables (Narayan \& Popham 1993). Alternatively, advection-domination may occur when the accretion rate is extremely high. In this case, the optical depth of the flow becomes so large that the radiation is unable to escape in less than a flow time. Popham et al. (1993) found that this may happen in FU Orionis systems. Advection-domination has been considered also in disks around supermassive black holes. Rees et al. (1982) discussed ion tori at very low accretion rates, while Begelman (1978) discussed radiation trapping at very high $\dot{M}$ where the photon diffusion time scale becomes longer than the radial inflow time scale (or advection time scale).

If advection-dominated conditions are present over a sufficiently wide range of radius in an accreting source, then we may expect the flow to approximate the self-similar solutions discussed here (cf, Fig. 1 in NY). The various properties of these solutions then become relevant. To summarize:

1. The flow is definitely not disk-like in morphology. In fact, the closest analog to our solutions in the accretion literature is Bondi (1952) spherical accretion. However, our flows differ in important ways from the Bondi problem. The gas in our solutions does rotate, it has non-trivial viscous interactions through which angular momentum is transported outward, and it has energy dissipation just as in ordinary accretion disks.

2. The angular velocity is significantly sub-Keplerian and this may have important implications for the spin-up of accreting stars. Stars which spin up through an advection-dominated mode of accretion are likely to reach a steady state with a rotation rate much below the "break-up limit." This may be one solution to the angular momentum problem which is discussed frequently in the context of star formation.

3. The radial accretion velocity is typically high in advection-dominated flows. Roughly, the velocity scales as $v \sim \alpha c_{s}$. In a cooling-dominated disk, $c_{s} \ll v_{f f}$, where $v_{f f}$ is the free-fall velocity, and so $v \ll v_{f f}$. However, advection-dominated flows have $c_{s} \sim v_{f f}$ and so for a reasonable $\alpha \sim 0.1$, we find $v \sim 0.1 v_{f f}$.

4. The Bernoulli parameter is positive over most of the flow (except the regions very close to the inner edge), and there is also likely to be violent convection especially close to the rotation axis. A fairly substantial bipolar outflow is quite likely under these conditions provided only that the material can remain adiabatic during the acceleration phase of the outflow.

5. The convective motions will transport both energy and angular momentum and will be a source of viscosity. This has the advantage that, even if for some reason the Balbus \& Hawley (1991) instability were to be ineffective, convective viscosity can still keep the accretion going.

6. By definition, advection-dominated accretion systems are under-luminous relative to the mass accretion rate. This is because the energy is carried along with the accreting gas as heat instead of being radiated. If the accreting object is a regular star, this energy must finally be radiated from the star and will be seen as stellar emission. However, if the accretor is a black hole, then most of the accretion energy can disappear through the horizon. It would be very misleading to estimate the mass accretion rate of such a system from the observed luminosity. 
7. The spectrum of an advection-dominated flow is likely to be quite different from that of a cooling-dominated accretion disk. If the flow is in the low $\dot{M}$ optically thin limit, then the temperature of the emitted radiation will be close to virial and the spectrum will be unusually hard. This appears to be the situation in cataclysmic variable boundary layers for $\dot{M} \lesssim 10^{-9.5} M_{\odot} \mathrm{yr}^{-1}$ (Narayan \& Popham 1993). In the opposite radiation-trapped limit at high $\dot{M}$, the photosphere will be much farther out than usual and the spectrum will be unusually soft. This is apparently the case in FU Orionis systems (Popham et al. 1993). Similar effects must be present in accreting neutron star and black hole systems, but this remains to be investigated.

We conclude with a final speculative comment on accretion disk coronae. An interesting feature of our solutions in the limit of large $\epsilon^{\prime}$ (efficient cooling) is that they have low density hot gas on top of an equatorial thin disk (see the $\epsilon^{\prime}=10$ solution in Fig. 1). The hot gas resembles the ad hoc coronae that various researchers have invoked in models of accretion disks. We speculate (i) that coronae are a natural and inevitable feature of any thin disk, (ii) that such coronae are best described as advection-dominated flows rather than as static atmospheres, and (iii) that these coronae will themselves have outflows for the reasons discussed in this paper, though with much smaller mass loss rates than in fully advection-dominated flows. Whenever a disk plus corona structure is formed, the accreting material must be divided in some self-consistent manner between the thin cool disk and the thick hot corona. One possibility is that the corona always has just enough mass in it to be marginally advection-dominated, i.e. to have $f_{\text {corona }} \sim 1 / 2$. Detailed calculations with radiative transfer are needed to confirm whether such a model will be self-consistent.

Acknowledgements: We thank Mitch Begelman and Henk Spruit for useful comments. This work was supported in part by the NSF under grant AST-9148279. 


\section{APPENDIX A: Comparison with Begelman and Meier (1982)}

Begelman \& Meier (1982, hereafter BM) considered a problem very similar to the one described in this paper, and it is useful to compare the two approaches. $\mathrm{BM}$ restricted their attention to a radiation-dominated accretion flow which is fully advection-dominated. In the language of this paper, their model corresponds to the particular case, $\gamma=4 / 3, \epsilon^{\prime}=\epsilon=1$. They considered however a more general viscosity model than we did, viz.

$$
\nu \propto p^{\alpha^{\prime}} \rho^{\beta^{\prime}} r^{\gamma^{\prime}} g(\theta)
$$

where $\alpha^{\prime}, \beta^{\prime}, \gamma^{\prime}$ are three arbitrary exponents. $g(\theta)$ is a general function of $\theta$, though in actual practice, BM set $g(\theta)=$ constant in their calculations. By imposing additional requirements like self-similarity, BM restricted their viscosity model to satisfy

$$
\beta^{\prime}=-\alpha^{\prime}, \quad 1+\alpha^{\prime}-\gamma^{\prime}=1 / 2,
$$

so that they had effectively a one-parameter family of viscosity models parametrized by $\alpha^{\prime}$. Our model of viscosity, eq. (2.6), corresponds to the particular choice,

$$
\alpha^{\prime}=1, \quad \beta^{\prime}=-1, \quad \gamma^{\prime}=3 / 2,
$$

and is one member of this family. We have also carried out a few calculations with another model corresponding to $\alpha^{\prime}=1 / 2, \beta^{\prime}=-1 / 2, \gamma^{\prime}=1$.

Starting with the basic equations $(2.7)-(2.10)$ which we have written in sec. 2, BM assumed that

$$
v_{r}, v_{\theta} \ll v_{\phi},
$$

and derived the following second-order differential equation for $\Omega(\theta)$,

$$
\begin{gathered}
\left(1-\sin ^{2} \theta \Omega^{2}\right)\left(\Omega^{\prime \prime}+3 \cot \theta \Omega^{\prime}-\frac{3}{4} \Omega\right)=\frac{\mu \sin ^{2} \theta \Omega}{2(\mu+4)}\left(\frac{9}{4} \Omega^{2}+\Omega^{12}\right) \\
-2\left(\beta^{\prime}+1\right) \sin ^{2} \theta \Omega^{\prime 2}-\left(\gamma^{\prime}+3 \beta^{\prime}+4\right) \cos \theta \sin \theta \Omega^{2} \Omega^{\prime}
\end{gathered}
$$

The parameter $\mu$ is the radial exponent of the pressure, $p \propto r^{\mu}$. For self-similar solutions, $\mu=-5 / 2$. Notice that eq. (A5) is only a second-order differential equation, whereas the system of equations we derived in this paper is sixth-order. The simplification arises because of the assumption $v_{r} \ll v_{\phi}$ (eq. A4).

It is straightforward to derive an equation similar to (A5) starting with our equations (2.16) - (2.19). As discussed in the paper, our solutions have $v \sim \alpha$. Therefore the condition $v \ll v_{\phi}$ is equivalent to the assumption $\alpha \ll 1$. In fact, all we need is $\alpha^{2} \ll 1$. Under this condition, we can neglect all terms involving $v^{2}$ and $\alpha v$ in eqs. (2.16), (2.17) and (2.19). Eq. (2.16) then gives

$$
c_{s}^{2}=\frac{2}{5}\left(1-\sin ^{2} \theta \Omega^{2}\right)
$$


while (2.17) gives

$$
\frac{d}{d \theta}\left(\rho c_{s}^{2}\right)=\cos \theta \sin \theta \rho \Omega^{2}
$$

Eliminating $v$ between (2.18) and (2.19) and substituting (A6) and (A7) we then find

$$
\begin{gathered}
\left(1-\sin ^{2} \theta \Omega^{2}\right)\left(\Omega^{\prime \prime}+3 \cot \theta \Omega^{\prime}-\frac{3}{4} \Omega\right)=-\frac{5}{6 \epsilon^{\prime}} \sin ^{2} \theta \Omega\left(\frac{9}{4} \Omega^{2}+\Omega^{\prime 2}\right) \\
-\frac{5}{2} \cos \theta \sin \theta \Omega^{2} \Omega^{\prime} .
\end{gathered}
$$

This is exactly equivalent to eq. (A5) provided we set $\epsilon^{\prime}=1$ (radiation-dominated, full advection) and $\beta^{\prime}=-1, \gamma^{\prime}=3 / 2$ (eq A3), $\mu=-5 / 2$. We thus find perfect agreement between our equations and those of BM so long as $\alpha^{2}, v^{2} \ll 1$.

All the solutions we have presented in this paper have $\alpha=0.1$ or smaller, so that $\alpha^{2} \ll 1$. Furthermore, all of our solutions have well-behaved non-singular $v(\theta)$ and satisfy $v^{2} \ll 1$ at all $\theta$. Therefore, although our solutions were obtained by solving the exact sixth-order equations (2.16) - (2.19), they do in fact satisfy BM's second-order equation (A5) very accurately. These solutions seem to have been missed by BM.

The solutions that BM described in their paper correspond to flows which extend from the equatorial plane at $\theta=\pi / 2$ to a free surface at $\theta=\theta_{F}$, inside of which is an empty funnel. A somewhat disturbing feature of their solutions is that the radial velocity $v$ diverges at $\theta=\theta_{F}$. The divergence arises because the ram pressure term $v^{2} / 2$ and the poloidal viscous term $(d / d \theta)(\alpha d v / d \theta)$ in eq. (2.16), which would normally control the divergence, were eliminated through the assumption $v_{r} \ll v_{\phi}$. A divergent solution is of course not compatible with the original assumption $v \ll 1$, and this implies an inconsistency in the solutions. Another odd feature of the BM solutions is that for fixed values of the parameters $\left(\epsilon^{\prime}, \alpha, \alpha^{\prime}, \beta^{\prime}, \gamma^{\prime}, \mu\right)$, they find a continuous family of self-similar solutions, with a continuously tunable funnel opening angle $\theta_{F}$. We find this infinity of solutions somewhat disturbing and worry that perhaps a boundary condition at the free surface may have been missed. We note that in our calculations, when we fix $\alpha$ and $\epsilon^{\prime}$, we find a unique fundamental solution. (We do have the curious higherorder solutions described in $\S 3.1$, where $\Omega(\theta)$ has one or more nodes, but these solutions still form only a discrete set, not the continuous family that BM find.)

We feel that it is important to search for solutions with empty funnels and free surfaces using the full sixth-order set of equations (2.16) - (2.19). The numerical methods which we have employed in the calculations described in this paper are incapable of finding such solutions. It is therefore a completely open question whether or not such solutions exist. Tori and empty funnels have been much discussed in accretion astrophysics, expecially in the context of AGN (see Frank, King \& Raine 1992 for a review). However, except for the work of BM, there have been no self-consistent dynamical models of such flows which include viscosity fully. In our opinion this is an important problem. 


\section{APPENDIX B: Non-Rotating Spherical Inflow}

In addition to the rotating solutions which are the main focus of the paper, the self-similar equations (2.16)-(2.19) allow a second non-rotating spherically symmetric branch of solutions which is closely related to the Bondi (1952) problem. To derive this solution we set $\Omega=0$ and $d / d \theta=0$. Equations (2.17) and (2.18) then immediately drop out. Equation (2.19) gives

$$
-\frac{3 \epsilon^{\prime} v}{2 \alpha}=3 v^{2},
$$

which has two solutions for $v$. One of these is $v=0$, which is the condition satisfied at $\theta=0$ by all the rotating solutions described in the paper. For spherical inflow, we consider the second possibility, namely

$$
v=-\frac{\epsilon^{\prime}}{2 \alpha} .
$$

Substituting this in eq (2.16), we then solve for the sound speed, to obtain

$$
c_{s}^{2}=\frac{2-\epsilon^{\prime 2} / 4 \alpha^{2}}{5-\epsilon^{\prime}} .
$$

Equations (B2), (B3) coupled with the self-similar scalings completely describe this branch of solutions. We see that these solutions are allowed whenever $\left|\epsilon^{\prime}\right|<$ $2 \sqrt{2} \alpha$ (or $\epsilon^{\prime}<5$ if $\alpha$ is large). Thus, a self-similar form of spherical accretion is possible so long as $|\gamma-5 / 3| \lesssim \alpha$.

Note that in pure Bondi (1952) spherical flow, a self-similar form of accretion or outflow is allowed only for a single value of $\gamma$, viz. $\gamma=5 / 3$. In contrast, we find that self-similarity is possible in the present problem for a range of values of $\gamma$. The additional freedom arises because we have viscosity. Even though the flow is not rotating, it still has a velocity divergence and this gives rise to the viscous dissipation term $3 v^{2}$ in the right of eq (B1). When $\gamma \neq 5 / 3$, self-similarity requires a radial gradient in the entropy (as discussed in §3.1). The solution feeds this gradient by tuning the magnitude of $v$ so as to supply the required energy input through viscous dissipation.

The other interesting feature is that we can have either self-similar spherical inflow or outflow, depending on the sign of $\epsilon^{\prime}$ (eq B2). Since viscosity is always a source of energy, the entropy has to increase in the direction of the flow. For $\gamma<5 / 3$, the entropy increases inward in the self-similar solution, and so the motion is also inwards, i.e. we have accretion. However, for $\gamma>5 / 3$, the solution corresponds to entropy increasing outward. In this case, therefore, our solution corresponds to a spherical self-similar wind.

Note that the spherical solutions described here may be either subsonic or supersonic. For $\epsilon^{\prime}$ close to zero, $v \ll c_{s}$, and we have subsonic conditions. However, as $\left|\epsilon^{\prime}\right| \rightarrow 2 \sqrt{2} \alpha$, the radial velocity increases and the sound speed decreases and we can have supersonic flows.

As a final comment, we recall that the rotating solutions described in the main text of the paper exist only for $\epsilon^{\prime}>C \alpha^{2}$ where $C$ is a constant of order unity $(\S 3.1)$. On the other hand, the spherical solutions described here exist for

$\epsilon^{\prime}<2 \sqrt{2} \alpha$. Thus, for $C \alpha^{2}<\epsilon^{\prime}<2 \sqrt{2} \alpha$ both solution branches are allowed. What is the relationship between the two solutions? Which solution will an actual flow prefer? Perhaps the answer is determined by the initial conditions of the flow, especially the angular momentum of the accreting gas. 


\section{References}

Abramowicz, M., Czerny, B., Lasota, J. P., \& Szuszkiewicz, E. 1988, ApJ, 332, 646

Balbus, S. A. \& Hawley, J. F. 1991, ApJ, 376, 214

Begelman, M. C. 1978, MNRAS, 184, 53

Begelman, M. C. \& Meier, D. L. 1982, ApJ, 253, 873

Bondi, H. 1952, MNRAS, 112, 195

Dubrulle, B. 1992, A\&A, 266, 592

Eggum, G. E., Coroniti, F. V., \& Katz, J. I. 1988, ApJ, 330, 142

Frank, J., King, A., \& Raine, D. 1992, Accretion Power in Astrophysics (Cambridge, UK: Cambridge Unniversity Press)

Hawley, J. F., Gammie, C. F. \& Balbus, S. A. 1994, preprint

Henriksen, R. N. \& Valls-Gaboud, D. 1994, MNRAS, 266, 681

Landau, L. D. \& Lifshitz, E. M. 1959, Fluid Mechanics (London: Pergamon Press)

Liang, E. P. 1988, ApJ, 334, 339

Lynden-Bell, D. \& Pringle, J. E. 1974, MNRAS, 168, 603

Mihalas, D. \& Mihalas, B. W. 1984, Foundations of Radiation Hydrodynamics (New York: Oxford University Press)

Narayan, R., Loeb, A., \& Kumar, P. 1994, ApJ, 431, 359

Narayan, R. \& Popham, R. 1993, Nature, 362, 820

Narayan, R. \& Yi, I, 1994, ApJ, 428, L13

Popham, R., Narayan, R., Hartmann, L., \& Kenyon, S. 1993, ApJ, 415, L127

Press, W. H., Teukolsky, S. A., Vetterling, W. T., \& Flannnery, B. P. 1992, Numerical Recipes (Cambridge: Cambridge University Press)

Rees, M. J., Begelman, M. C., Blandford, R. D., \& Phinney, E. S. 1982, Nature, 295,17

Ryu, D., \& Goodman, J. 1992, ApJ, 388, 438

Shakura, N. I. \& Sunyaev, R. A. 1973, A\&A, 24, 337

Spruit, H. C., Matsuda, T., Inoue, M., \& Sawada, K. 1987, MNRAS, 229, 517 


\section{Figure Captions}

Figure 1. Self-similar solutions corresponding to $\alpha=0.1, \epsilon^{\prime}=0.1,1,10$. Top left: angular velocity $\Omega$ as a function of polar angle $\theta$. Top right: radial velocity $v$. Bottom left: density $\rho$. Bottom right: square of the sound speed, $c_{s}^{2}$.

Figure 2. Isodensity contours in the meridional plane for four solutions. The top two panels and the bottom left panel correspond to the solutions shown in Figure 1. The bottom right panel shows a solution in which $\Omega$ reverses sign twice (see text).

Figure 3. Comparison of the exact solutions of this paper with the height- integrated solutions of NY. The solid lines correspond to the height-integrated solutions; the dotted lines correspond to spherical averages (cf eq. 3.5); the dashed lines correspond to cylindrical $z$-averages (eqs. 3.6-3.8); and the long-dashed lines correspond to midplane values. The height-integrated values agree remarkably well with the spherical averages for all values of $\epsilon^{\prime}$.

Figure 4. Dimensionless Bernoulli parameter $b$ as a function of the spherical polar angle $\theta$ for self-similar solutions with $\alpha=0.1, \epsilon=0.333$ (i.e. $\gamma=1.5$ ), and from bottom to top, $\epsilon^{\prime}=300,100,10,1,0.75,0.33$ (i.e. $f=0.0011,0.0033,0.033$, $0.33,0.4465,1)$. Note that the Bernoulli parameter is always positive close to the rotation axis, and is positive at all $\theta$ for $\epsilon^{\prime}<0.75$ (i.e. $f>0.446$ ).

Figure 5. The ratio between the convection term and advection term in the energy equation, shown as a function of $\theta$, for a solution with $\alpha=0.01, \alpha_{c}=0.005$, $\epsilon=0.01, f=1$. For all $\theta>\theta_{\text {crit }}=33^{\circ}$, the convection term is smaller than the advection term and self-similar advection-dominated accretion is possible. For $\theta<\theta_{\text {crit }}$, convection dominates over advection and we speculate that convection will initiate a bipolar outflow in this region of the flow. Note from Fig. 4 that this region has the most positive Bernoulli parameter and is therefore most susceptible to being ejected. 
This figure "fig1-1.png" is available in "png" format from: http://arxiv.org/ps/astro-ph/9411058v1 
This figure "fig2-1.png" is available in "png" format from: http://arxiv.org/ps/astro-ph/9411058v1 
This figure "fig1-2.png" is available in "png" format from: http://arxiv.org/ps/astro-ph/9411058v1 
This figure "fig2-2.png" is available in "png" format from: http://arxiv.org/ps/astro-ph/9411058v1 
This figure "fig1-3.png" is available in "png" format from: http://arxiv.org/ps/astro-ph/9411058v1 